\title{
Asymptomatic Professional Footballers: Prevalence of Ankle Retinacula Injury with Associated Lateral Ligament and Tendon Abnormalities
}

\author{
K. Win ${ }^{1}$, M. Gillett' ${ }^{2}$ R. Botchu ${ }^{5}$ A.K. Suokas ${ }^{3}$, A. Seth ${ }^{4}$, S.L. James ${ }^{5}$ \\ 1 Birmingham City Football Club, Cattell Road, Birmingham, United Kingdom \\ 2 Nottingham Forest Football Club, The City Ground, Nottingham, United Kingdom \\ 3 Research and Teaching Centre, The Royal Orthopaedic Hospital NHS Foundation Trust, Bristol Road South, \\ Northfield, Birmingham, United Kingdom \\ ${ }_{4}$ Sports and Exercise Medicine, The Royal Orthopaedic Hospital NHS Foundation Trust, Bristol Road South, \\ Northfield, Birmingham, United Kingdom \\ ${ }^{5}$ Department of Musculoskeletal Radiology, The Royal Orthopaedic Hospital NHS Foundation Trust, Bristol Road \\ South, Northfield, Birmingham, United Kingdom
}

\section{CORRESPONDING AUTHOR:}

Rajesh Botchu

Department of Musculoskeletal Radiology

The Royal Orthopedic Hospital

Bristol Road South

Northfield, Birmingham, United Kingdom

E-mail: drbrajesh@yahoo.com

DOI:

10.32098/mltj.03.2020.22

LEVEL OF EVIDENCE: 4

\section{SUMMARY}

Background. The retinacula are important dynamic stabilizers around the ankle joint and are susceptible to potential injury in high-level footballers.

Aim. The purpose of this study was to evaluate the prevalence of retinacula injury in asymptomatic professional football players and for the presence of associated ankle tendon or ligament abnormalities.

Methods. Seventeen professional football players from one English premier league club underwent ultrasound examination of both ankles. The retinacula around the ankle were measured for thickness and compared with normal values. Ankle tendons were evaluated for the presence of abnormal vascularity or tendinopathy, tendon sheath effusions, and for peroneal tendon subluxation. Lateral ankle ligaments were also examined.

Results. The results indicated increased thickness of the retinacula at the following sites: superior extensor retinaculum on the medial side $(1.5 \mathrm{~mm}$, normal $0.9 \mathrm{~mm})$; superior peroneal retinaculum at the anterior insertion $(2.16 \mathrm{~mm}$, normal $1 \mathrm{~mm})$; and flexor retinaculum at the anterior insertion into the tibia $(1.78 \mathrm{~mm}$, normal. $0.9 \mathrm{~mm})$. Fluid around the tibialis posterior tendon was a frequent finding representing $74 \%$, whilst over $25 \%$ revealed fluid within the peroneal tendon sheaths. Over $50 \%$ of the scans revealed thickened and abnormal lateral ligament complexes.

Conclusions. The thickening of the retinacula may indicate scarring from previous injury or repetitive micro-trauma, attributable to the ball kicking biomechanics, kinematics and contact injuries during tackles. Furthermore tendon sheath fluid was commonly seen on both the medial and lateral side of the ankle. We conclude that chronic scarring and thickening of these structures should be recognized as a common and often incidental finding and the significance in asymptomatic professional footballers is minimal.

\section{KEY WORDS}

Ankle; football; retinaculum; tendon; ligament. 


\section{BACKGROUND}

Soccer (football) is the world's most popular sport, with an estimated 270 million people (roughly $4 \%$ of world's population) actively playing or officiating in the game (1).

At a professional level, high intensity bursts of activity on top of 90 minutes of lower intensity aerobic effort are required with players covering an average distance of $10 \mathrm{~km}$ during a 90 min match (2). Union of European Football Associations (UEFA) data indicates that ankle injuries account for 10 to $18 \%$ of all injuries in high-level soccer (2) with an incidence of $0.7 / 1000$ hour of exposure (3). A professional squad of 25 players is predicted to see an average of $4-5$ ankle sprains during a season (3). The most commonly reported mechanism of injury is a tackle on the medial or lateral side of the weight bearing limb (4) with foul play involved in $40 \%$ of match-related ankle sprains (3). Despite an anticipated quick return to sport after ankle sprains (mean lay-off 15 days) (3), previous authors have shown that up to $80 \%$ of athletes still present with persistent symptoms at 1.5 to 4 year follow up (5-9).

The foot and ankle are a complex anatomical area. Movement in the sagittal plane occurs at the talo-crural joint with dorsi-flexion and plantar-flexion, whilst movement in the coronal plane occurs at the sub-talar joint with inversion and eversion. (10) Active movement is dependent on contraction of the long flexor and extensor muscles of the lower leg, with their tendons undergoing significant angulation and directional changes whilst traversing the ankle joint to insert into the tarsal and meta-tarsal bones. They are held in place by the retinacula of the ankle, which prevent them from a form of subluxation referred to as bowstringing. The retinacula are localized thickenings of the crural fascia and typically heal with fibrosis and scarring after injury. When the muscles contract, the retinacula permit the tendons to glide longitudinally whilst maintaining approximation to the underlying bones, thus augmenting the mechanical strength and stability of the tendon complexes.

Given that ankle ligament injuries are common in professional soccer, accounting for $51-81 \%$ of all football related ankle injuries (3), our hypothesis was that evidence of a previous retinacula injury should be frequently identified. The aim of this study was to identify the prevalence of abnormal thickening of the retinacula structures of the ankle in asymptomatic professional soccer players during a screening ultrasound examination. The lateral ligament complex as well as the anterior, medial and peroneal tendons was also examined to assess for the prevalence of associated soft tissue abnormalities.

The extensor retinacula is derived from the superficial crural aponeurosis of the leg and serves as a restraint to the extensor tendons at the front of the ankle joint and dorsum of the foot. It can be divided into the superior extensor and inferior extensor retinacula.

The superior extensor retinaculum is a transverse rectangular aponeurotic band that originates from the anterior border of the fibula and lateral malleolus, crossing over the anterior aspect of the ankle just above the tibio-talar joint to insert into the anterior tibial crest and medial malleolus (11). Deep to it are the extensor tendons (medial to lateral) tibialis anterior, extensor hallucis longus, extensor digitorum longus and peroneus tertius, the dorsalis pedis vessels and the deep peroneal nerve. It is reported that in $25 \%$ of cases, the superficial and deep layer fibers form a separate tunnel for the tibialis anterior tendon (12).

The inferior extensor retinaculum is a complex $\mathrm{X}$ or $\mathrm{Y}$ shaped structure situated at the anterior aspect of ankle and the dorsum of the foot. Previous studies have reported that this structure is difficult to visualize completely on MRI and ultrasound (11), hence it was not included in the ultrasound protocol used in this study.

\section{The peroneal retinacula include the superior and inferior peroneal retinaculum}

The superior peroneal retinaculum is a transverse rectangular fibrous band located at the lateral aspect of the ankle. It attaches to the lateral border of the retro-malleolar groove proximally and the lateral wall of the calcaneus distally. It covers the peroneus brevis and peroneus longus tendons when they pass through the retro-malleolar groove to enable functional gliding movements within the groove. It is well documented as a primary restraint for peroneal tendon subluxation and also serves as a secondary restraint to ankle inversion and lateral ankle instability (12). A fibrous triangular cartilage is commonly seen at its distal attachment on the peroneal periosteum.

The inferior peroneal retinaculum is an oblique band, connecting the lateral rim of the sinus tarsi to the lateral calcaneus below the trochlear process. The flexor retinaculum is a triangularly shaped fibrous aponeurosis that extends from the tip of the medial malleolus to the medial calcaneal process. It restrains the flexor tendons (anterior to posterior) tibialis posterior, flexor digitorum longus and flexor hallucis longus.

\section{METHODS}

Local ethical committee approval as well approval from Director of performance of the club was obtained for the study (13). The sample comprised of the first team professional players at a single Premier League soccer club. Players were only included in the study if both their ankles were 
asymptomatic at the time of examination and they were participating in full unrestricted training. Additionally, if players had undergone previous foot or ankle surgery then they were excluded from the study in order to avoid bias of the retinacula measurements undertaken.

The study consisted of 17 male professional soccer players. The average age was 28 years (range 20-34 years), 11 were right foot and 6 left foot dominant.

Screening medical examinations are common in professional sport and the ultrasounds undertaken for the purposes of this study formed part of an existing and ongoing player screening program which encompasses both clinical and imaging assessment. The ultrasound screening program is approved by the club's governance committee to allow prospective data collection in asymptomatic players.

Ultrasound scanning of both ankles (total 34 scans) was carried out by a fellowship trained specialist musculoskeletal radiologist. The scans were performed with a Siemens Acuson P300 ultrasound system and a linear high frequency probe.

In addition to the retinacula measurements, the ultrasound screening protocol also included examination of the peroneal, flexor and extensor tendons, together with evaluation of the lateral ligament structures reliably visualised with ultrasound namely the anterior inferior tibio-fibular, anterior talo-fibular and calcaneo-fibular ligaments. The tendons were evaluated for the presence of abnormal vascularity/ tendinopathy, tendon sheath effusions/tenosynovitis and peroneal tendon subluxation. The lateral ligaments were examined for their integrity and any abnormal thickening indicative of prior injury.

Normal reference values for retinacula structures were compared to the players' measurements. (14) Reference values

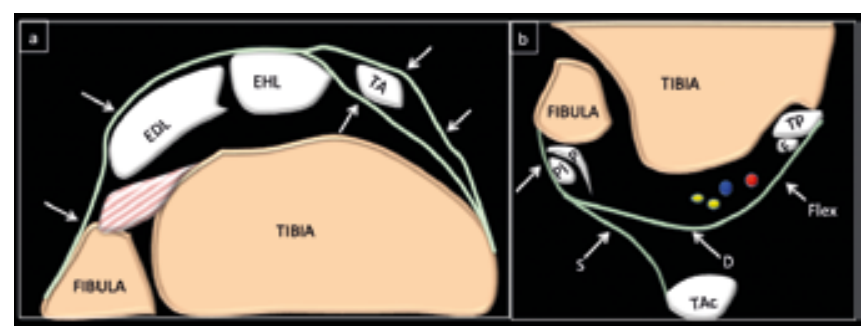

Figure 1. Animations of axial section of $15 \mathrm{~mm}$ proximal to ankle joint (a) showing attachment of superior extensor retinaculum (arrow), (b) showing the superior peroneal retinaculum (arrow) continuous with superficial (S) and deep aponeurosis (D) of the posterior ankle and continuous with superior part of the flexor retinaculum. TA (tibialis anterior), EHL (extensor hallucis longus), EDL (extensor digitorum longus), P (peroneus brevis, PI (peroneus longus), TAc (tendoachilles), TP (tibialis posterior), $\mathrm{F}$ (flexor digitorum longus). were obtained for eight retinacula structures as follows. The mean thickness of the superior extensor retinaculum was $0.9 \mathrm{~mm}$ and the range $0.7-1.3 \mathrm{~mm}$ (14) (figures 1,2). Measurements for thickness were taken at both the anterior and posterior aspects of the superior peroneal retinaculum, and the mean thickness was $1.0 \mathrm{~mm}$ and the range 0.7 $2.6 \mathrm{~mm}$ (14). The mean thickness of the inferior peroneal retinaculum was $0.8 \mathrm{~mm}$ and the range $0.7-1.0 \mathrm{~mm}$ (14). An anterior and posterior measurement of flexor retinaculum thickness was made, and the mean thickness was $0.9 \mathrm{~mm}$ and the range $0.7-1.0 \mathrm{~mm}$ (14) (figures 2,3,4).

The mean and range of the thickness of the retinacula were calculated for each of the chosen eight structures. The sample mean and the reference value were compared separately for the dominant and non-dominant foot using the one sample t-test. Shapiro-Wilks tests indicated that the data were not normally distributed, but the one sample $\mathrm{t}$-test was used, as it is fairly robust to departures of normality. P-values $<0.05$ were reported as significant. Measurements from each site were plotted and presented with reference values (mean and range) to show the distribution of values. Statistical analysis was carried out using SPSS 22 (IBM Corporation, NY, USA).

\section{RESULTS}

The superior extensor retinaculum demonstrated a mean thickness of $1.57 \mathrm{~mm}$ (range $0.9-2.5 \mathrm{~mm}$ ) medially and $0.89 \mathrm{~mm}$ (range $0.5-1.9 \mathrm{~mm}$ ) laterally. Compared to the reference value, the mean thickness of the medial aspect of the retinaculum was statistically significantly larger for both dominant and non-dominant foot (table I). The difference was not statistically significant on the lateral side (figures 5,6)

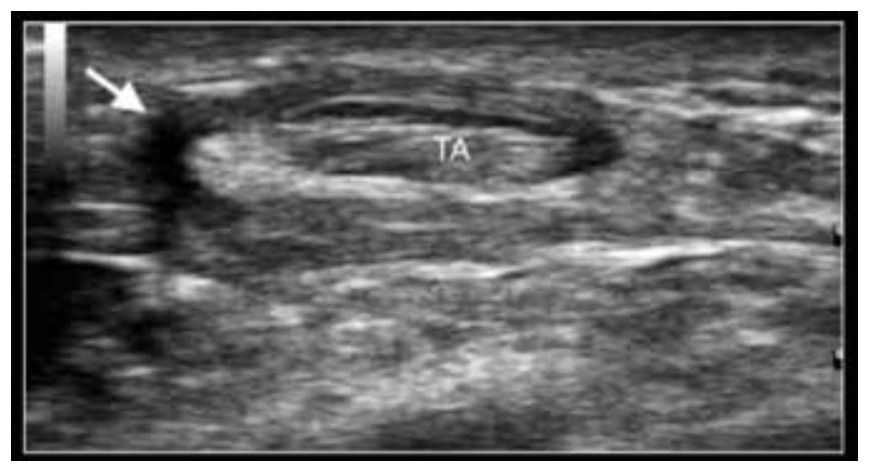

Figure 2. Transverse image demonstrating a separate tibialis anterior (TA) tunnel. The medial aspect of the superior extensor retinaculum is identified curving around the medial border of the TA tendon (white arrow) and passing deep to the tendon. The retinaculum is seen coursing over the superficial aspect of TA. 


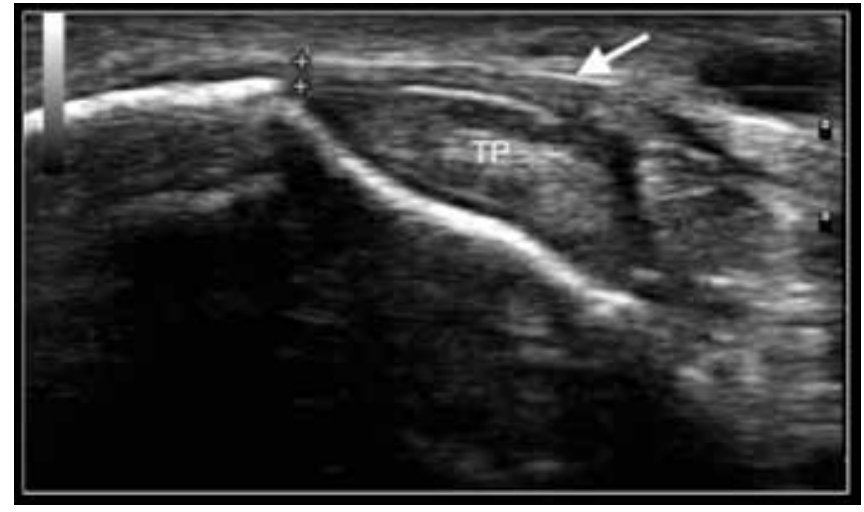

Figure 3. Transverse image illustrating a normal flexor retinaculum arising from the tibia medially (white arrow). The site of medial measurement is shown with the tibialis posterior tendon (TP) being identified deep to the retinaculum.

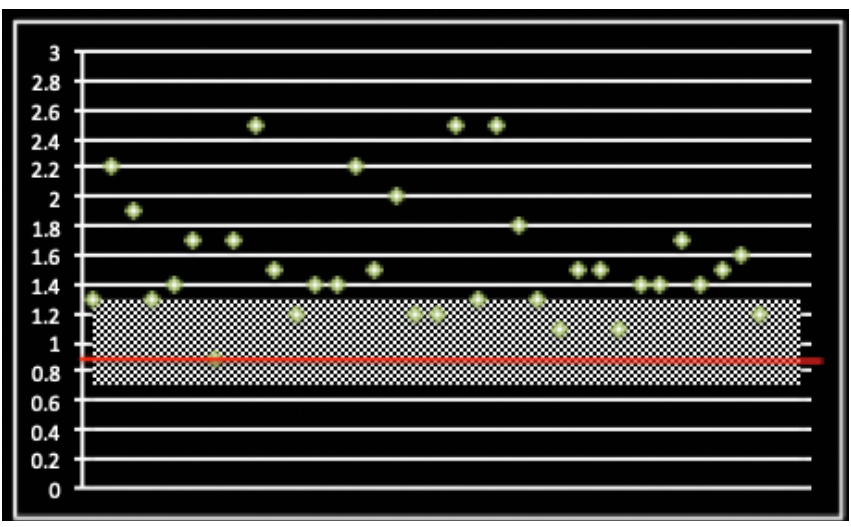

Figure 5. Medial measurement of the superior extensor retinaculum ( $n=34$ scans). The shaded area represents the normal reference values (mean $0.9 \mathrm{~mm}$; range $0.7-1.3 \mathrm{~mm}$ ).

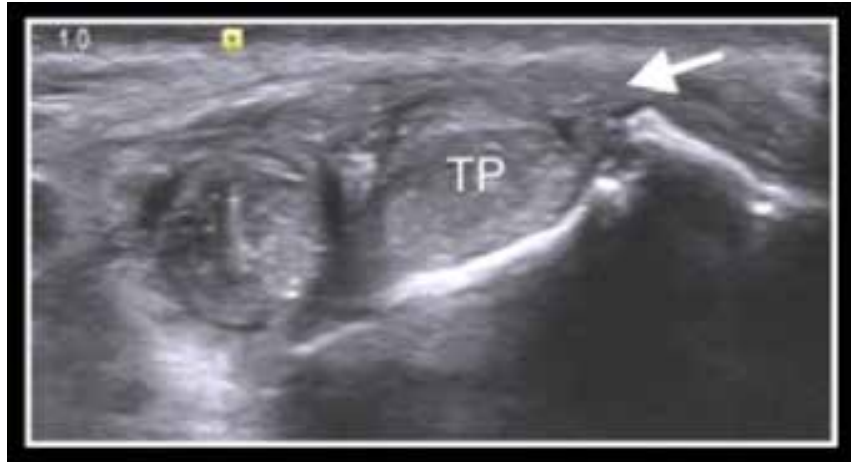

Figure 4. Transverse image demonstrating a thickened flexor retinaculum arising from the tibia (white arrow). There is also an area of bony irregularity associated with the tibial cortex. The tibialis posterior tendon is again illustrated (TP).

Table I. One sample t-test comparing the sample mean with the reference value for each retinacula structure by dominant vs. non-dominant foot.

\begin{tabular}{lll}
\hline & Dominant foot & Non-dominant foot \\
\hline Superior extensor retinaculum: medial & $\mathrm{t}=6.189, \mathrm{p}<0.001^{*}$ & $\mathrm{t}=7.181, \mathrm{p}<0.001^{*}$ \\
\hline Superior extensor retinaculum: lateral & $\mathrm{t}=-0.143, \mathrm{p}=0.888$ & $\mathrm{t}=0.036, \mathrm{p}=0.972$ \\
\hline Superior peroneal retinaculum: anterior & $\mathrm{t}=5.227, \mathrm{p}<0.001^{*}$ & $\mathrm{t}=6.403, \mathrm{p}<0.001^{*}$ \\
\hline Superior peroneal retinaculum: posterior & $\mathrm{t}=1.450, \mathrm{p}=0.167$ & $\mathrm{t}=0.975, \mathrm{p}=0.344$ \\
\hline Inferior peroneal retinaculum: anterior & $\mathrm{t}=-1.628, \mathrm{p}=0.123$ & $\mathrm{t}=-1.369, \mathrm{p}=0.190$ \\
\hline Inferior peroneal retinaculum: posterior & $\mathrm{t}=-1.186, \mathrm{p}=0.253$ & $\mathrm{t}=-0.466, \mathrm{p}=0.647$ \\
\hline Flexor retinaculum: anterior & $\mathrm{t}=5.762, \mathrm{p}<0.001^{*}$ & $\mathrm{t}=5.851, \mathrm{p}<0.001^{*}$ \\
\hline Flexor retinaculum: posterior & $\mathrm{t}=1.255, \mathrm{p}=0.227$ & $\mathrm{t}=2.223, \mathrm{p}=0.041^{* *}$ \\
\hline
\end{tabular}

* p-values $<0.05$ indicate statistically significant difference

$* *$ Significant association was caused by an outlier.

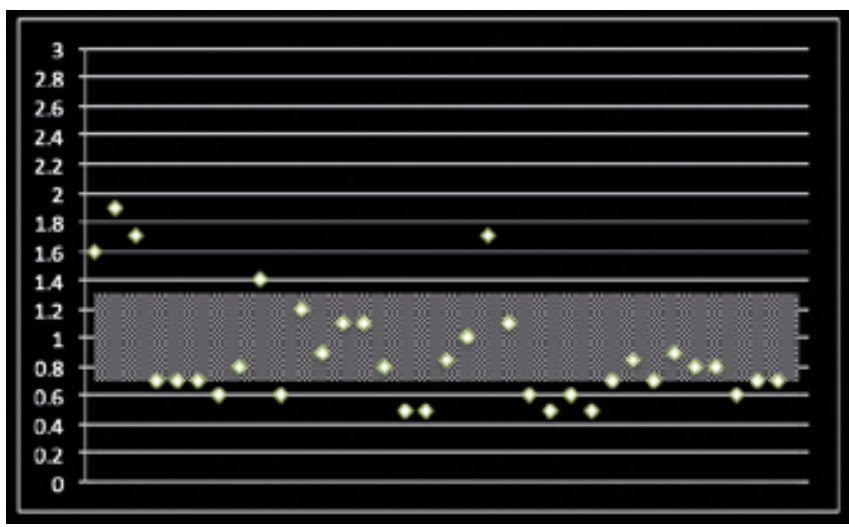

Figure 6. Lateral measurement of the superior extensor retinaculum ( $n=34$ scans). The shaded area represents the normal reference values (mean $0.9 \mathrm{~mm}$; range $0.7-1.3 \mathrm{~mm}$ ). 
11 out of 34 ankles (29\%) had a separate tunnel for the tibialis anterior tendon. There was one player, who had a separate tendon tunnel in his dominant leg and 5 players with bilateral separate tibialis anterior tunnels.

The superior peroneal retinaculum demonstrated a mean thickness of $2.16 \mathrm{~mm}$ (range $0.7-4.4 \mathrm{~mm}$ ) anteriorly and $1.18 \mathrm{~mm}$ (range $0.6-3.6 \mathrm{~mm}$ ) posteriorly. Compared to the reference value, the mean thickness of the anterior side of the retinaculum was statistically significantly larger for both dominant and non-dominant foot (table I). The difference was not statistically significant on the posterior side. (figures $\mathbf{7 , 8 )}$ Two ankles, which were completely asymptomatic, demonstrated peroneal tendon dislocation on dynamic ultrasound examination with the foot dorsiflexed and everted implying disruption of the superior peroneal retinaculum.

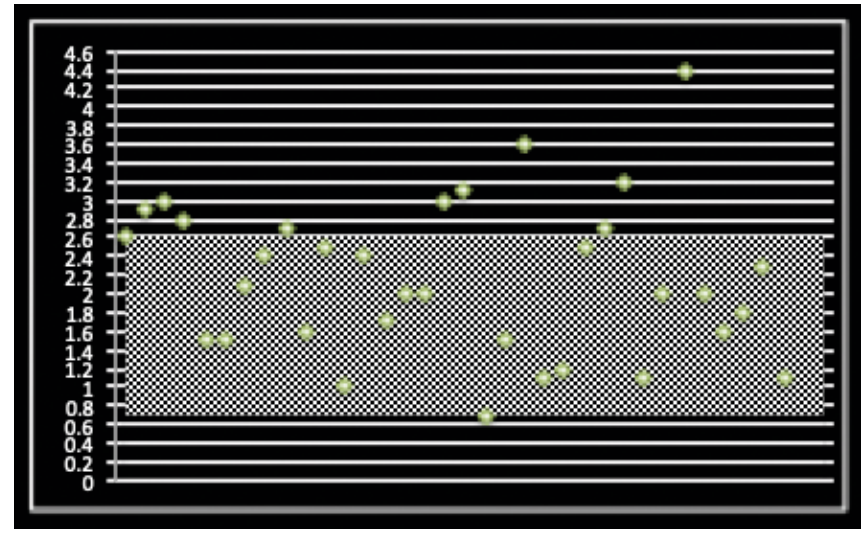

Figure 7. Anterior measurement of the superior peroneal retinaculum ( $n=34$ scans). The shaded area represents the normal reference values (mean $1.0 \mathrm{~mm}$; range $0.7-2.6 \mathrm{~mm}$ ).

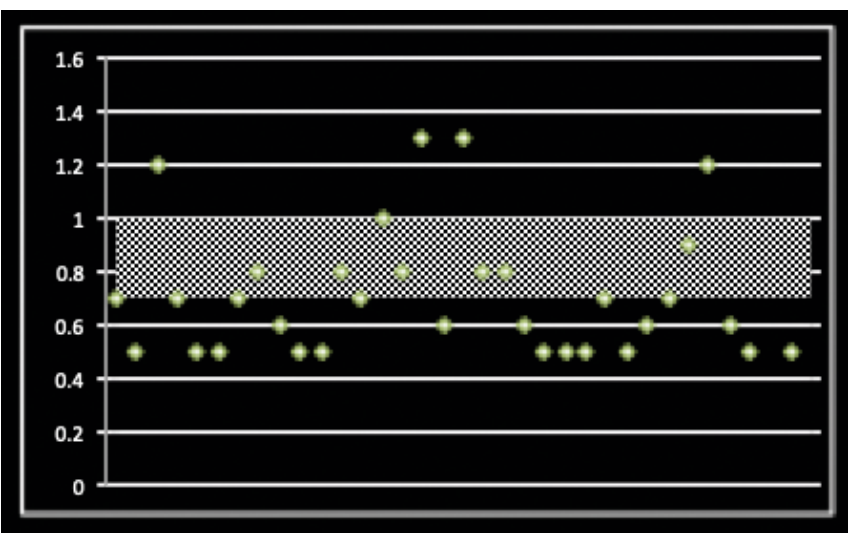

Figure 9. Anterior measurement of the inferior peroneal retinaculum ( $n=34$ scans). The shaded area represents normal reference values (mean 0.8; range 0.7 - $1.0 \mathrm{~mm}$ ).
The inferior peroneal retinaculum demonstrated a mean thickness of $0.71 \mathrm{~mm}$ (range $0.5-1.3 \mathrm{~mm}$ ) anteriorly and $0.74 \mathrm{~mm}$ (Range $0.4-1.4 \mathrm{~mm}$ ) posteriorly. The mean thickness was not statistically significantly different from the reference value for any of the retinacular structures (table I) (figures 9,10).

The flexor retinaculum demonstrated a mean thickness of $1.78 \mathrm{~mm}$ (range $0.8-3.6 \mathrm{~mm}$ ) anteriorly and $1.06 \mathrm{~mm}$ (range 0.6-2.5 $\mathrm{mm}$ ) posteriorly. Compared to the reference value, the mean thickness of the anterior side of the retinaculum was statistically significantly larger for both dominant and non-dominant foot (table I). On the posterior side, the difference was only statistically significant for the non-dominant foot. However, this significant result was created by an outlier $(2.5 \mathrm{~mm}$ ) (figures 11,12).

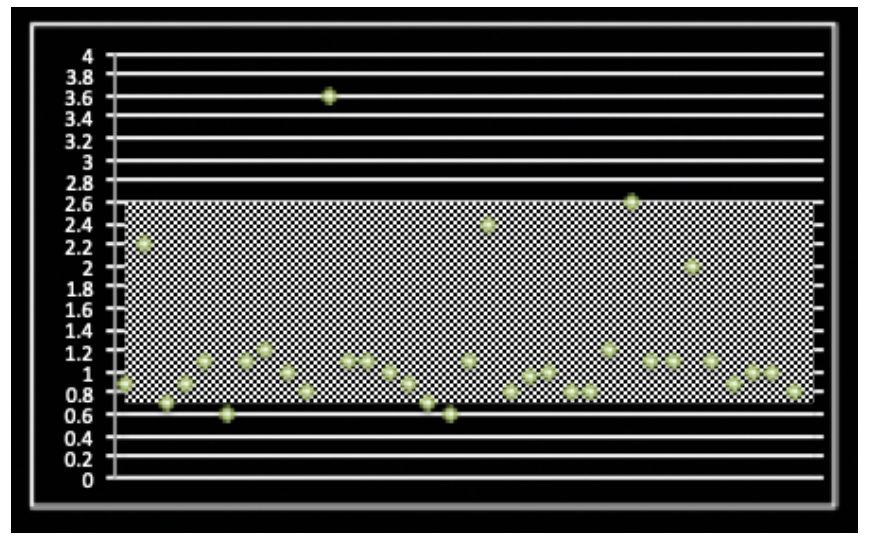

Figure 8. Posterior measurement of the superior peroneal retinaculum ( $n=34$ scans). The shaded area represents the normal reference values (mean $1.0 \mathrm{~mm}$; range $0.7-2.6 \mathrm{~mm}$ ).

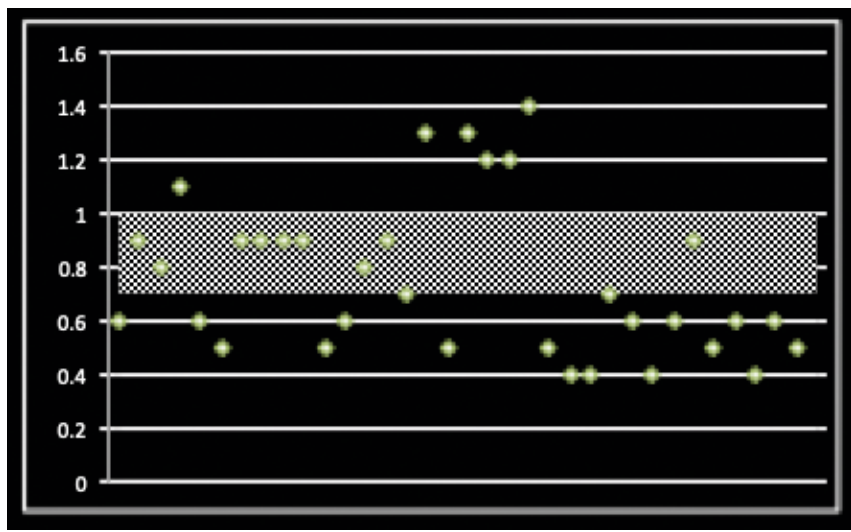

Figure 10. Posterior measurement of the inferior peroneal retinaculum ( $n=34$ scans). The shaded area represents normal reference values (mean $0.8 \mathrm{~mm}$; range $0.7-1.0 \mathrm{~mm}$ ). 


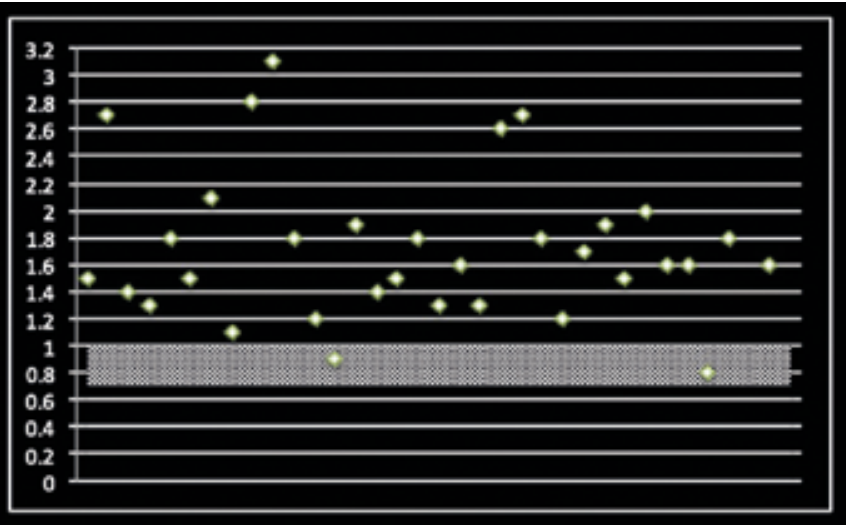

Figure 11. Anterior measurement of the flexor retinaculum (n $=34$ scans). The shaded area represents the normal reference values (mean $0.9 \mathrm{~mm}$; range $0.7-1 \mathrm{~mm}$ ).

25 out of 34 ankles (74\%) elicited fluid within the tibialis posterior tendon sheath. Only one out of these 25 ankles demonstrated increased vascularity around an area of thickened synovium. One player was found to have a low-grade partial tear of the tibialis posterior tendon with mixed intrasubstance change indicating associated tendinopathy at the level of the medial malleolus in his dominant foot.

The ultrasound appearance of the tendons of flexor hallucis longus and flexor digitorum longus was normal in most players. Only 3 out of 34 ultrasounds showed fluid around the flexor hallucis longus tendon, whilst a completely different set of 3 players had a tendon sheath effusion in flexor digitorum longus.

4 out of $34(12 \%)$ demonstrated tendon sheath effusions around the tibialis anterior tendons, of which, 2 were associated with separate tibialis anterior tunnels. No significant tendon sheath effusion or tendinopathy was identified in extensor hallucis longus and extensor digitorum longus.

Scanning of the peroneal longus tendon revealed a tendon sheath effusion in 9 out of 34 ankles $(26 \%)$. This finding was not associated with neovascularisation. A similar result was found in the peroneal brevis tendon, with 10 scans (29\%) demonstrating fluid in the tendon sheath.

In this study population, thickening of the lateral ligaments was a common finding. 76\% (26/34) revealed a thickened AITFL. However only 2 out of these 26 were associated with neovascularization. $71 \%(24 / 34)$ of scans elicited an abnormal ATFL, with 16 demonstrating a thickened scarred ligament and 8 showing a deficient and attenuated ATFL.

A thickened CFL was seen in $56 \%$ of ankle scans (19/34), with one third of players (6/17) demonstrating bilateral ankle involvement.

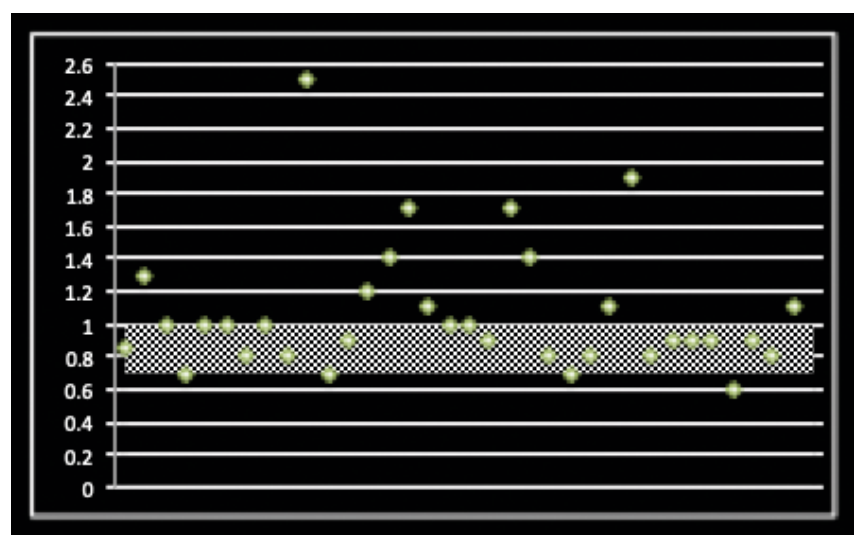

Figure 12. Posterior measurement of the flexor retinaculum ( $n=34$ scans). The shaded area represents the normal reference values (mean $0.9 \mathrm{~mm}$; range $0.7-1 \mathrm{~mm}$ ).

\section{DISCUSSION}

This is the first study to examine the ultrasound appearances of the ankle retinacula in asymptomatic professional soccer players. Our principle findings suggesting increased thickness of the retinacula on the following sites: superior extensor retinaculum on the medial side, superior peroneal retinaculum on the anterior side and flexor retinaculum on the anterior side of the ankle.

Asymptomatic tendon sheath effusions are most commonly identified within the tibialis posterior $(74 \%)$ and to a lesser extent in the peroneus longus $(26 \%)$ and brevis tendon $(29 \%)$ sheaths. Lateral ligament complex injuries are also a common associated finding with the majority of players showing ultrasound evidence of prior injury to the AITFL, ATFL and CFL.

We propose the following two mechanisms as contributing factors to explain the radiological appearances observed in this study:

direct compression of the retinacula can occur during a ball control and kicking manoeuvre, or from contact with another player;

in soccer, significant force is applied through the foot and ankle whilst controlling, passing or blocking the ball using the medial or lateral side of the foot and ankle (15). The repeated sub-maximal stress on normal tissue could induce local tissue inflammation and scar tissue formation in the long-term and may explain the pattern of chronic retinacula scarring observed in this study.

Among different types of ball kicks, the instep kick is normally used for the generation of fast ball speed (16). During ball strike, professional players keep the ankle locked in plantar flexion in order to maximize the force 
generated to propel the ball (17). A study conducted by Tol et al. (18) that evaluated the relationship of the kicking action in soccer to ankle impingement is highly pertinent to this study. In 39\% of kicking actions, the dynamic plantar flexion angle recorded on impact exceeded the maximum static angle. This study also concluded that over $75 \%$ of ball strikes involved ball contact with the medial malleolus and the base of the $1^{\text {st }}$ metatarsal. This area is in close anatomical proximity to the medial attachments of the superior extensor retinaculum and the flexor retinaculum, which were found to be significantly thickened in this study. Hence an ankle at the extreme range of plantar flexion contacts the ball at speed on the medial aspect of the foot and ankle, creating an impact force in the region of 1025 Newtons (18). Given the repeated execution of this skill by a professional footballer in both training and matches, the repetitive strain on the antero-medial ankle structures is likely to be significant and a potential cause of retinacula thickening.

Player to player contacts during tackles can result in significant force transmission through the foot and ankle region, which may traumatize both the antero-lateral and medial aspects of the ankle joint $(4,19)$. However, traumatic contacts in professional soccer do not occur with the same frequency as repetitive kicking activity, and it is the authors' hypothesis that this mechanism is likely to be less significant to our findings than repetitive kicking.

High-level soccer places extreme musculo-skeletal demands on the body, especially in the lower limbs. Multidirectional movement patterns including jumping, landing, twisting, turning and cutting are common and induce high forces around the ankle joint (20). The repetitive loading of the foot and ankle during the functional movements of soccer may contribute to the chronic scarring of the retinacula observed around the ankle.

We hypothesized that retinacula injuries may be associated with injuries to the lateral ligament complex.

The most common mechanism of ankle injury in soccer is excessive inversion with plantar flexion, which often leads to lateral ligament complex injuries (20). The lateral ligament injuries are often depicted as isolated injuries to the anterior talofibular ligament (ATFL) and calcaneofibular ligament (CFL). Despite early return to sport, the rate of re-injury and chronic instability are as high as $80 \%$ in athletes $(5-9,21)$. Previous literature infers that symptoms such as pain, giving way and reduced ankle range of motion can often persist following acute ankle ligamentous injury(5-9,22-24). In one study (8) $32 \%$ of 648 patients attending hospital with an ankle inversion injury reported chronic pain, swelling or recurrent injury 7 years after their initial presentation (5). In this ultrasound-based study, we have collected evidence that injury of the lateral ligament is a common finding in soccer players who are clinically asymptomatic. These athletes are still able to function at a high athletic level and hence the significance of this finding in isolation is debatable. A variety of biomechanical changes to the sub-talar and inferior tibio-fibular joints have been shown to follow lateral ligament injury including increased anterior translation and internal rotation of the talus $(25,26)$, excessive pronation and sub-talar laxity (25). Given the extent, variety and frequency of these findings, it is entirely possible that other ankle stabilizing structures are injured at the same time as the lateral ligament and this list may include the ankle retinacula.

At the present time there is inadequate evidence in the medical literature to definitively associate lateral ligament complex injury with retinacula injury. However, in the authors' experience, when ligamentous injuries are imaged in the acute phase oedema is frequently visualized around the retinacula located adjacent to the injured ligaments. It is possible that the thickening of the anterior side of the superior peroneal retinaculum observed in this study is commonly associated with lateral ligament injury and it would be helpful to evaluate this possibility with acute injuries in the future.

The common mechanisms of acute traumatic retinacula injury have been reported in previous studies $(11-14,27)$. An acute injury of the extensor retinaculum is observed with traumatic, forceful dorsiflexion of the foot. Sudden forceful plantar flexion and eversion can cause tearing of the flexor retinaculum, which may be associated with tibialis posterior tendon dislocation (28). Acute traumatic injuries of the extensor and flexor retinacula are infrequent (11) and it is considered unlikely that any of the subjects included in this study sustained a significant acute injury of either of these structures previously.

The peroneal retinaculum serves as a secondary restraint of ankle inversion movement $(12,29)$. An injury of the superior peroneal retinaculum can occur when dorsiflexion of the ankle is coupled with forceful eversion from peroneal muscle contraction $(11,12,29)$. Both peroneal retinaculum injuries and peroneal tendon subluxation are commonly linked to chronic lateral ankle instability $(12,12,29)$. Previous literature suggests that non-operative management of peroneal tendon dislocation can lead to recurrent subluxation of the peroneal tendons, with primary surgical repair recommended for individuals with high athletic demands (29). In our study, 2 players were found to have peroneal retinaculum injuries associated with peroneal tendon dislocations. Interestingly both players were asymptomatic and remained fully functional in professional soccer, challenging the necessity for immediate repair in this patient group.

Tendon sheath effusions were found in $74 \%$ of tibialis posterior tendons, $12 \%$ of tibialis anterior tendons, $26 \%$ 
of peroneus longus tendons and $29 \%$ of peroneus brevis tendons. Only one of these tendons, a tibialis posterior tendon with a partial tear and intrasubstance change suggestive of tendinopathy, demonstrated imaging evidence of further pathology.

Hence on the basis of this study, tendon sheath effusions can be considered a normal finding in professional footballers and are likely to be reactive and potentially a further consequence of the repeated kicking nature of the sport.

Bianchi and colleagues had described the sonographic features of normal retinacula around the ankle and their pathologies but the study population was not confined to footballers as our study (30).

There are a number of limitations of this study. First and foremost, we are assuming that the reference ranges reported in the cadaveric study reflect an accurate representation of normal retinacula thickness. We do not believe this represents a significant drawback as if there had been previous injuries to the cadaver group this would tend to cause an underestimation of injury prevalence in the screened professional soccer players. The sample size is small as the squad size of football team is limited. We have tried to postulate

\section{REFERENCES}

1. http://www.fifa.com/worldfootball/bigcount/index.html.

2. http://www.uefa.com/newsfiles/156571.

3. Walden M, Hagglund M, Ekstrand J. Time-trends and circumstances surrounding ankle injuries in men's professional football: an 11-year follow-up of the UEFA Champions League injury study. Br J Sports Med 2013;47(12):748-53 doi: 10.1136/ bjsports-2013-092223.

4. Giza E, Fuller C, Junge A, Dvorak J. Mechanisms of foot and ankle injuries in soccer. Am J Sports Med 2003;31(4):550-4.

5. Anandacoomarasamy A, Barnsley L. Long term outcomes of inversion ankle injuries. Br J Sports Med 2005;39(3):e14; discussion e14 doi: 10.1136/bjsm.2004.011676.

6. Gerber JP, Williams GN, Scoville CR, Arciero RA, Taylor DC. Persistent disability associated with ankle sprains: a prospective examination of an athletic population. Foot Ankle Int 1998;19(10):653-60.

7. Green T, Refshauge K, Crosbie J, Adams R. A randomized controlled trial of a passive accessory joint mobilization on acute ankle inversion sprains. Phys Ther 2001;81(4):984-94.

8. Konradsen L, Bech L, Ehrenbjerg M, Nickelsen T. Seven years follow-up after ankle inversion trauma. Scand J Med Sci Sports 2002;12(3):129-35.

9. BL B. Effects of ankle sprain in a general clinical population 6 to18 months after medical evaluation. Arch Fam Med 1999;8:143-8.

10. Brockett CL, Chapman GJ. Biomechanics of the ankle. Orthop Trauma. 2016;30(3):232-238. doi:10.1016/j.mporth. 2016.04.015. potential mechanisms for our findings and this is based on previous literature descriptions of injury mechanisms rather than direct biomechanical analysis. A single experienced radiologist made the ultrasound measurements and there is the potential for inter-observer variability in measurements. Finally, we are also assuming that MRI and ultrasound measurements can be used interchangeably when making comparisons between the cadaver and professional soccer readings.

\section{CONCLUSIONS}

Asymptomatic retinacula injuries are common in professional soccer. Despite chronic scarring of the retinacula, the players remain able to function at high level with no reported symptoms. Imaging evidence of lateral ligament complex injury is a common finding as is the presence of fluid within the tendon sheaths of the ankle tendons.

\section{CONFLICT OF INTERESTS}

The authors declare that they have no conflict of interests.

11. Demondion X, Canella C, Moraux A, Cohen M, Bry R, Cotten A. Retinacular disorders of the ankle and foot. Semin Musculoskelet Radiol 2010;14(3):281-91 doi: 10.1055/s0030-1254518.

12. Geppert MJ, Sobel M, Bohne WH. Lateral ankle instability as a cause of superior peroneal retinacular laxity: an anatomic and biomechanical study of cadaveric feet. Foot Ankle 1993;14(6):330-4.

13. Padulo J., Oliva F., Frizziero A., Maffulli N. Muscles, Ligaments and Tendons Journal - Basic principles and recommendations in clinical and field Science Research: 2018 update. MLTJ 2018; 8(3): 305 - 307.

14. Numkarunarunrote N, Malik A, Aguiar RO, Trudell DJ, Resnick D. Retinacula of the foot and ankle: MRI with anatomic correlation in cadavers. AJR Am J Roentgenol 2007;188(4):W348-54 doi: 10.2214/AJR.05.1066.

15. Madden CC. Netter's sports medicine. Philadelphia: Saunders/Elsevier, 2010.

16. Levanon J, Dapena J. Comparison of the kinematics of the full-instep and pass kicks in soccer. Med Sci Sports Exerc 1998;30(6):917-27.

17. Barfield WR, Kirkendall DT, Yu B. Kinematic instep kicking differences between elite female and male soccer players. J Sports Sci Med 2002;1(3):72-9.

18. Tol JL, Slim E, van Soest AJ, van Dijk CN. The relationship of the kicking action in soccer and anterior ankle impingement syndrome. A biomechanical analysis. Am J Sports Med 2002;30(1):45-50. 
19. Hamilton WG, Geppert MJ, Thompson FM. Pain in the posterior aspect of the ankle in dancers. Differential diagnosis and operative treatment. J Bone Joint Surg Am 1996;78(10):1491-500.

20. Kofotolis ND, Kellis E, Vlachopoulos SP. Ankle sprain injuries and risk factors in amateur soccer players during a 2-year period. Am J Sports Med 2007;35(3):458-66 doi: 10.1177/0363546506294857.

21. Smith RW, Reischl SF. Treatment of ankle sprains in young athletes. Am J Sports Med 1986;14(6):465-71.

22. Gehring D, Faschian K, Lauber B, Lohrer H, Nauck T, Gollhofer A. Mechanical instability destabilises the ankle joint directly in the ankle-sprain mechanism. Br J Sports Med 2014;48(5):377-82 doi: 10.1136/bjsports-2013-092626.

23. Gillman SF. The impact of chiropractic manipulative therapy on chronic recurrent lateral ankle sprain syndrome in two young athletes. J Chiropr Med 2004;3(4):153-9 doi: 10.1016/ S0899-3467(07)60103-7.

24. Loudon JK, Reiman MP, Sylvain J. The efficacy of manual joint mobilisation/manipulation in treatment of lateral ankle sprains: a systematic review. Br J Sports Med 2014;48(5):36570 doi: 10.1136/bjsports-2013-092763.
25. Denegar CR, Miller SJ, 3rd. Can Chronic Ankle Instability Be Prevented? Rethinking Management of Lateral Ankle Sprains. J Athl Train 2002;37(4):430-35.

26. Wainright WB, Spritzer CE, Lee JY, et al. The effect of modified Brostrom-Gould repair for lateral ankle instability on in vivo tibiotalar kinematics. Am J Sports Med 2012;40(9):2099-104 doi: 10.1177/0363546512454840.

27. Hatch GF, Labib SA, Rolf RH, Hutton WC. Role of the peroneal tendons and superior peroneal retinaculum as static stabilizers of the ankle. J Surg Orthop Adv 2007;16(4):187-91.

28. Sharma R, Jomha NM, Otto DD. Recurrent dislocation of the tibialis posterior tendon. Am J Sports Med 2006;34(11):1852-4 doi: 10.1177/0363546506288729(publi shed Online First: Epub Date).

29. Maffulli N, Ferran NA, Oliva F, Testa V. Recurrent subluxation of the peroneal tendons. Am J Sports Med 2006;34(6):986-92 doi: 10.1177/0363546505283275.

30. Bianchi S, Becciolini M. Ultrasound Features of Ankle Retinacula: Normal Appearance and Pathologic Findings. J Ultrasound Med. 2019;38(12):3321-3334. 